University of Nebraska - Lincoln

DigitalCommons@University of Nebraska - Lincoln

\title{
An Analytical Model for Virtual Topology Reconfiguration in Optical Networks and A Case Study
}

\author{
Xi Yang \\ University of Nebraska-Lincoln, xyang@cse.unl.edu \\ Byrav Ramamurthy \\ University of Nebraska-Lincoln, bramamurthy2@unl.edu
}

Follow this and additional works at: https://digitalcommons.unl.edu/cseconfwork

Part of the Computer Sciences Commons

Yang, Xi and Ramamurthy, Byrav, "An Analytical Model for Virtual Topology Reconfiguration in Optical Networks and A Case Study" (2002). CSE Conference and Workshop Papers. 116.

https://digitalcommons.unl.edu/cseconfwork/116

This Article is brought to you for free and open access by the Computer Science and Engineering, Department of at DigitalCommons@University of Nebraska - Lincoln. It has been accepted for inclusion in CSE Conference and Workshop Papers by an authorized administrator of DigitalCommons@University of Nebraska - Lincoln. 


\title{
An Analytical Model for Virtual Topology Reconfiguration in Optical Networks and A Case Study*
}

\author{
Xi Yang and Byrav Ramamurthy \\ Department of Computer Science and Engineering \\ University of Nebraska-Lincoln \\ Lincoln, NE 68588-0115, U.S.A.
}

\begin{abstract}
An analytical model for Virtual Topology Reconfiguration (VTR) in optical networks is developed. It aims at the optical networks with a circuit-based data plane and an IPlike control plane. By identifying and analyzing the important factors impacting the network performance due to VTR operations on both planes, we can compare the benefits and penalties of different VTR algorithms and policies. The best VTR scenario can be adaptively chosen from a set of such algorithms and policies according to the real-time network situations. For this purpose, a cost model integrating all these factors is created to provide a comparison criterion independent of any specific VTR algorithm and policy. A case study based on simulation experiments is conducted to illustrate the application of our models.
\end{abstract}

\section{INTRODUCTION}

$\mathrm{N}$ backbone optical networks, the changing traffic patterns may devalue the optimization of the static virtual topology design. Rapid and flexible Virtual Topology Reconfiguration (VTR) is a highly desired feature for building the nextgeneration optical network control plane. The VTR problem is intractable due to both its computation complexity and online nature [1]. There were two directions in the literature to explore solutions. In one direction, the VTR problem was formulated using global optimization techniques, such as ILP, and solved using various heuristic algorithms $[1,2,3,4,5]$. In the other direction, studies were focused on the VTR policy, which is represented by the configuration of a set of VTRrelated network parameters. The performance gain and the cost penalty of VTR were studied to find out an optimal policy, which provides a good tradeoff $[6,7]$.

This study is motivated by the fact that none of the proposed VTR algorithms and policies is better than others for all network scales, topologies and traffic patterns. A practical control plane should not be bound to a fixed reconfiguration algorithm or policy. Instead, an intelligent VTR scenario can be used to decide for a specific situation the best choice from a set of available algorithms and policies. The basis of such a choice is through providing a precise model to analyze the performance gain and cost penalty of a candidate VTR heuristic algorithm or policy. However, none of the previous models is complete enough for such a purpose. In particular, the impact of VTR on the extensively favored IP-controlled optical networks has yet to be studied. Actually all the previous studies on VTR have focused on designing reconfiguration algorithms and policies. The modeling of VTR impact on the networks was only implicitly expressed in the optimization objectives and constraints $[1,2,3,4,5,6,7]$.

*This work was supported in part by NSF grants (ANI-0074121 and EPS-0091900).
Our study furthers the previous work on modeling the impact of VTR with two concerns. Firstly, the previous studies only handled the impact of reconfiguration on the data plane (also referred to as the transport plane in the literature). All previous modeling work on the performance metrics such as those of load balancing and delay and on the impact of lightpath disruption was based on analyzing the data plane. Our study reveals that the impact of reconfiguration on the control plane is also significant, especially when we are using an IP-like control on the optical networks. Secondly, to compare different VTR algorithms and policies and make choice adaptively, we need to model the common impact factors. Hence, in our study the important factors in both the data plane and the control plane are extracted and modeled without depending on a specific VTR algorithm or policy.

\section{ANALYTICAL MODELS}

In this section we identify and analyze the impact factors of VTR at the data and control planes in the optical networks.

\section{A. Network Model}

In this study, the next-generation optical network is modeled as the combination of a circuit-based data plane with an IP-like control plane.

The upper-layer service, carried by IP, ATM, Frame Relay or SONET traffic, are mapped to a fraction of bandwidth in a lightpath at the virtual topology layer. From the viewpoint of the control plane, all services in the data plane are circuits and can be explicitly routed. Upon the requests of circuit establishment, the bandwidth is allocated through reservations along a sequence of lightpaths from source to destination. Each lightpath can be viewed as a link in the network. Therefore, we have assumed a multi-hop virtual topology network. The routing of upper-layer traffic is based on the available bandwidth on the lightpaths of the existing virtual topology. When the load of the existing virtual topology becomes unbalanced, deletions and creations of lighpaths will be triggered.

In this study, we do not consider the requirement of wavelength continuity on each single lightpath. We also omit the effects due to network failure events and assume that all underlying physical devices will not change. Only traffic demands can directly affect the reconfiguration.

The traffic demands over the virtual topology are modeled using the source-destination traffic matrix. We consider an Nnode mesh network. By $l_{d}(t)$ we denote the number of logical links or lightpaths deleted at time t. By $\Delta$ we denote the average in-degree at each node. Multiple links are allowed 
between the neighboring nodes in the virtual topology. $V_{i j}(t)$ specifies the number of lightpaths between nodes $i$ and $j$, so the matrix of $V_{i j}(t)$ represents the virtual topology. $\Lambda_{s d}(t)$ denotes the traffic demand by bandwidth, whose unit is Mbps, where $s d$ is the source-destination pair. $\lambda_{i j}^{s d}(t)$ is the $0-1$ mapping variable that routes the traffic $\Lambda_{s d}(t)$ on $V_{i j}(t)$, where 1 means the traffic demand between the nodes $s$ and $d$ is routed on one of the lightpaths between the nodes $i$ and $j$, and 0 means otherwise. $\Delta t$ is defined as the time increment of each VTR step; so the virtual topology is changed from $\mathrm{V}_{i j}(t)$ to $\mathrm{V}_{i j}(t+\Delta t)$ while the traffic demands changes from $\Lambda_{s d}(t)$ to $\Lambda_{s d}(t+\Delta t)$ and the traffic routing from $\lambda_{i j}^{s d}(t)$ to $\lambda_{i j}^{s d}(t+\Delta t)$.

\section{B. Data Plane}

Two impact factors are introduced by VTR operations at the data plane. A better performance is the common goal of all existing VTR algorithms and policies. Despite the diverse objectives and metrics, viz. load balance, hop-distance and blocking probability, we can convert the performance gain of VTR into the cost saving in the network constructions and operations and hence extract the first common impact factor. The penalty of VTR at the data plane is due to lightpath disruption, which is also shared by all VTR algorithms and policies. Although the performance metrics can vary a lot, two of them are most widely adopted, i.e. the delay metric and the load balancing metric.

\section{1) Performance gain}

We define $\Phi\left(V_{i j}(t), t\right)$ as the performance objective

function at time $t$, which is determined by the configuration virtual topology at that time. When time advances by $\Delta t$, if the network does not reconfigure its virtual topology, its performance objective function is $\Phi\left(V_{i j}(t), t+\Delta t\right)$. Otherwise, in each VTR step, $\mathrm{V}_{i j}(t)$ is changed to $\mathrm{V}_{i j}(t+\Delta t)$. From the viewpoint of the upper layer, since the lightpaths are the links for upper-layer traffic, reconfiguration means changed connectivity, capacity and delay. Hence the performance objective function is recalculated as $\Phi\left(V_{i j}(t+\Delta t), t+\Delta t\right)$. In the data plane, the performance gain function is defined as

$$
\Phi\left(V_{i j}(t+\Delta t), t+\Delta t\right)-\Phi\left(V_{i j}(t), t+\Delta t\right)
$$

\section{2) Lightpath disruption}

In previous studies, the penalty of VTR was often referred to as the lightpath disruption due to reconfiguration operations, which delete some lightpaths while adding others. The deletions or disruptions of lightpaths lead to packet loss and service outage at the upper layer. One of the objectives of the proposed VTR algorithms is minimizing the number of such disruptions. We quantify the number of lightpath disruptions between the nodes $i$ and $j$ as

$$
U\left(V_{i j}(t)-V_{i j}(t+\Delta t)\right)
$$

where the function $U(x)$ is defined as $U(x)=x(x>0)$ or $0(x$ $\leq 0$ ). Each deletion of a lightpath between nodes $i$ and $j$, even if there still remains another lightpath between the nodes, incurs a penalty or cost, because refreshing the routing tables will take some time. We assume adding lightpaths does not disrupt the operation of existing lightpaths.

\section{Control Plane}

There has been increasing interests in designing the control plane of optical networks using the extensions of the existing IP control protocols based on the Generalized Multiprotocol Label Switching (GMPLS) developed by IETF, which is quite different from the traditional control and management plane of a circuit-based data transport network, such as that of SONET. However, none of the previous studies explicitly considered the impact of VTR on the control plane. In the IP-controlled optical networks, an IP-like topology discovery mechanism is used, which has immediate response to the changes in the underlying virtual topology. Following that, the routing tables have to be refreshed to reflect the changed topology. Another affected factor is the admission control, which is also sensitive to changes in the virtual topology. Hence the performance of the control plane is not immune to the impact of VTR. We measure the impact of these two factors using blocking probability, which will be justified later.

\section{1) Topology discovery}

The straightforward mapping of IP topology discovery mechanism onto the optical control plane is carried out by the automatic and distributed topology discovery. There have been several proposals and drafts by IETF and other organizations that suggest the OSPF Link State Packets (LSPs) or BGP advertisement messages should be extended to advertise the link states between the nodes in the optical layer [8]. Flooding or selective flooding mechanism is used to broadcast the link-state updates to all the nodes in the network. Hence any changes following a VTR operation will be automatically notified without a centralized control (see Fig. 2.1). This mechanism is more scalable and flexible. However, as shown by previous experiments, the convergence latency of the BGP topology discovery mechanism in the Internet can reach up to fifteen minutes [13].

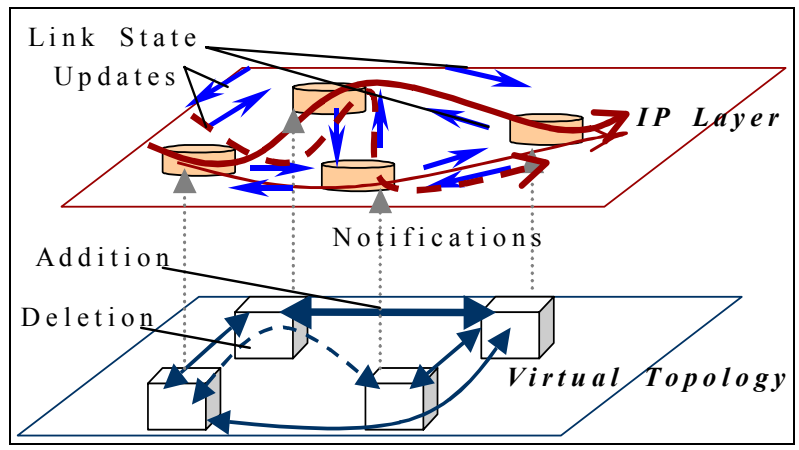

Fig. 2.1 Distributed Topology Discovery.

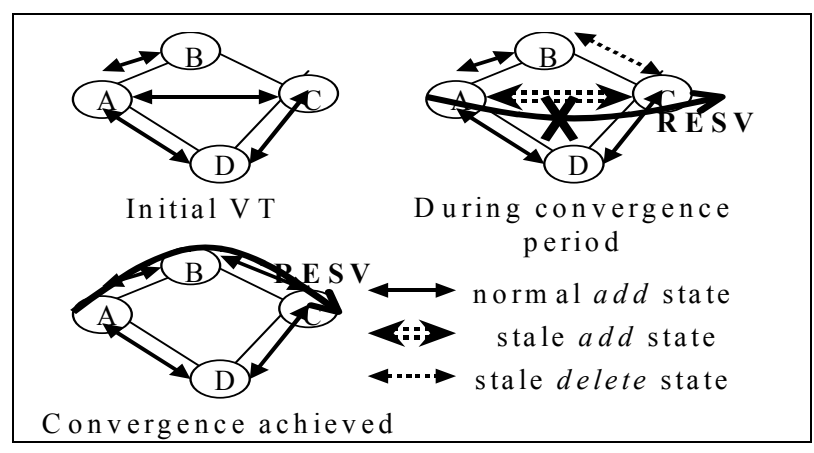

Figure 2.2. Blocking due to stale link states. 
Long convergence time leads to inconsistent or stale link states at some nodes and hence incorrect information about the network topology. As illustrated in Figure 2.2, the connection requests for bandwidth reservations on the existing lightpaths may be blocked due to stale link states at a node during the convergence period.

\section{2) Admission control}

Before global convergence is achieved, the edge nodes may send requests for bandwidth reservation on a sequence of lightpaths along the route found according to the old link states. When a reservation fails due to incorrect link states, i.e. lightpath states, this request is blocked. For example, if a lightpath is deleted but the link states at some nodes still indicate it is available, the bandwidth reservation on this lightpath will fail and the call request will be blocked (see Fig. 2.2). Added lighpaths are not considered because the incorrect link states of an added lightpath only make it unavailable but do not block the request. Since we have assumed upper-layer traffic is explicitly routed using a fraction of bandwidth reserved on a sequence of lightpaths, blocking a connection request means blocking all the traffic corresponding to this fraction of bandwidth. Hence, we can use blocking probability to measure the penalty or cost incurred on the control plane.

\section{3) Blocking probability}

As has been argued, the model of blocking probability consists of both the impact factors of topology discovery and admission control. We define the background average blocking probability as $\mathrm{P}_{0}$, which represents the blocking due to other factors except VTR. By $T_{\text {conv }}$, we denote the maximum convergence time. The blocking probability of a connection request is deduced as follows.

We assume a uniform source-destination traffic distribution. Shortest Path First (SPF) source routing is used. We do not consider the wavelength assignment problem. For the N-node network, the total number of links is $N \times \Delta$, where $\Delta$ is the average in-degree. The number of deleted links or lightpaths is $l_{d}(t)$. Immediately after the deletions, the topology discovery mechanism has not changed corresponding link states at any node. All arriving call requests are blocked if their routes are selected though a deleted link.

For a single-hop path, due to the assumption of the uniform source-destination traffic distribution, a fraction $\frac{l_{d}(t)}{N \Delta}$ of all the connection requests arriving immediately after the deletions are blocked due to the incorrect link state information about the deleted links.

Generally, for an h-hop path, we assume that all the deleted lightpaths have the equal probability to be independently selected to route through by an arriving call request. The blocking probability for this path is equal to

$$
P_{0}+\left(1-\left(1-\frac{l_{d}(t)}{N \Delta}\right)^{h}\right)\left(1-P_{0}\right)
$$

where $P_{0}$ is contributed by other factors while the rest is by VTR.

Note that the deleted lightpaths may have belonged to the same path before deletion. However, for the arriving connection requests, the assumption of independence for the selection of these lightpath into a new routing path is still reasonable. To be practical, the probability of the new paths routed through a deleted lightpath can be derived based on the actual virtual topology and traffic pattern at a specific time. As a theoretical model, we simply apply this equation to all multihop situations. We use the average weighted hop count $\mathrm{H}$ to substitute $h$ in equation (3) to get a uniform blocking probability for all arriving connections immediately after the deletions, which equals to $P_{0}+\left(1-\left(1-\frac{l_{d}(t)}{N \Delta}\right)^{H}\right)\left(1-P_{0}\right)$.

From the time $t_{0}$, when the deletions of lightpaths happen, the topology discovery mechanism begins to update the link states at all network nodes. Until $t_{0}+T_{\text {conv }}$, when all link states get converged, the blocking probability due to incorrect link state information used by the admission control shrinks to zero. We assume that, during the convergence period $\left(t_{0}, t_{0}+\right.$ $\left.T_{\text {conv }}\right)$, the fraction of the number of incorrect link states decreases from 1 to 0 following the function $e(t), 0<t \leq T_{\text {conv }}$. On average the blocking probability due to VTR at the control plane is proportional to the number of incorrect link states, and hence the equation of the blocking probability is revised as

$$
P_{0}+\left(1-\left(1-\frac{l_{d}(t)}{N \Delta}\right)^{H}\right)\left(1-P_{0}\right) e\left(t-t_{0}\right), t_{0}<t \leq t_{0}+T_{c o n v}
$$

\section{COST MODELS}

\section{A. Glossary}

$T_{\text {conv }}$, convergence time in seconds for topology discovery. $N$, number of network nodes.

$\Delta$, average number of in-degree of a node in the virtual topology.

$H$, average number of hops (or lightpaths) in a traffic path.

$\Phi\left(V_{i j}(t), t\right)$, network performance objective function at time $t$, using the virtual topology $V_{i j}(t)$.

$e(t)$, fraction of the number of incorrect link states relative to the number at the beginning of the topology discovery convergence period $\left(0, T_{\text {conv }}\right)$. It decreases from 1.0 to 0 .

$C_{e}$, integral of $e(t)$ (normalized to $T_{\text {conv }}$ ), a constant.

$S_{i j}(t)$, cost factor representing the service type carried at time $\mathrm{t}$ on the disrupted lightpaths between the nodes $i$ and $j$. Its value is a number no less than 1 .

$C_{a}$, average network revenue per second of the carrier. It is the unit for all the following cost definitions.

$C_{b}\left(t_{\text {dispt }}\right)$, cost of a lightpath disruption with the disruption time $t_{\text {disrpt }}$.

$C_{g}$, multiplier converting the performance gain to the negative cost, a constant.

$C_{D}(t+\Delta t)$, cost at the data plane in a VTR step $(t \rightarrow t+\Delta t)$.

$C_{C}(t+\Delta t)$, cost at the control plane in a VTR step. 
$C_{O}(t+\Delta t)$, sum of $C_{D}(t+\Delta t)$ and $C_{C}(t+\Delta t)$ in a VTR step.

$C_{P}(t+\Delta t)$, cost representing the negative of the performance gain in a VTR step.

$C_{A L L}(t+\Delta t)$, integrated cost, equal to the sum of $C_{P}(t+\Delta t)$ and $C_{O}(t+\Delta t)$ in a VTR step.

$C_{A L L}^{\prime}(t+\Delta t)$, revised integrated cost in a VTR step, using the average weighted hop count as performance metric.

\section{B. Data Plane}

The impact of lightpath disruptions dominates the VTR cost in the data plane. The next-generation backbone has to carry multiple types of services. For those mission-critical services, even a short period of disruption cannot be tolerated. However, there are also many IP-based services allowing relatively longer outage. According to [9], over $95 \%$ of the services can survive a $200 \mathrm{~ms}$ outage. Fig. 3.1.a shows the impact of the disruption in different time ranges (represented by rectangles in the figure) [10]. The longer the disruption, the more stages of time ranges are involved. Hence, we can formulate the cost of disruption as a step-wise function. We define the cost factor in the $l$-th range as $c_{l}$. Then the total cost factor for first $\mathrm{k}$ ranges is defined as $c(k)=\sum_{l=1}^{k} c_{l}$. To distinguish the disruption time with the time $t$ we have used previously, we let $t_{\text {disrpt }}$ denote the disruption time. We combine some time ranges, and define

$$
C_{b}\left(t_{\text {dispt }}\right)= \begin{cases}c(1), & t_{\text {disppt }} \leq 0.2 \mathrm{~s} \\ c(2), & t_{\text {dispt }} \leq 2 \mathrm{~s} \\ c(3), & t_{\text {dispt }} \leq 10 \mathrm{~s} \\ c(4), & t_{\text {disrpt }} \leq 300 \mathrm{~s} \\ c(5), & t_{\text {dispt }}>300 \mathrm{~s}\end{cases}
$$

which is shown in Fig. 3.1.b.

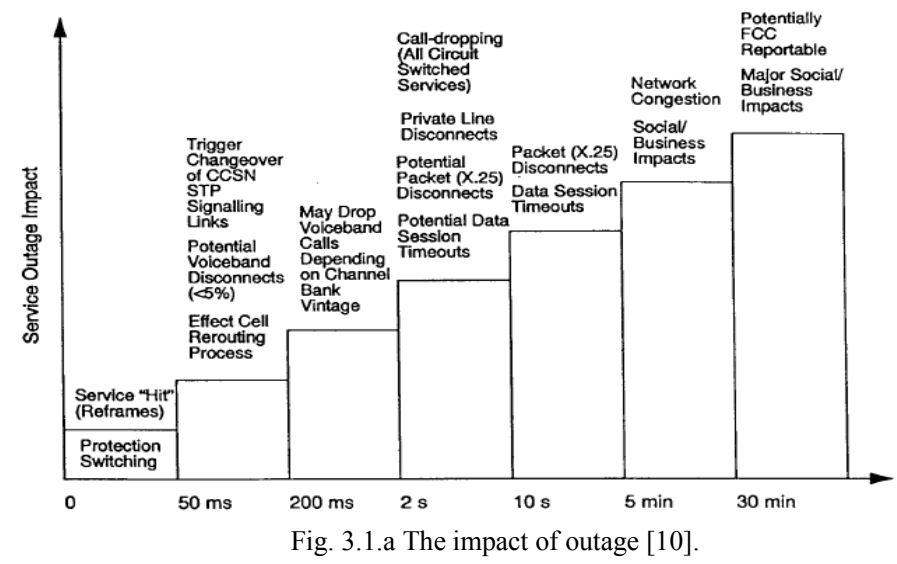

In addition to the time factor, the impact of lighpath disruptions also varies because the carried service types are different. We define the service-specific cost factor of the lightpaths between the nodes $i$ and $j$ as $S_{i j}(t)$. For normal Internet services, e.g. regular WWW and Email services, $S_{i j}(t)$ equals 1 . For other services, e.g. online banking, $S_{i j}(t)$

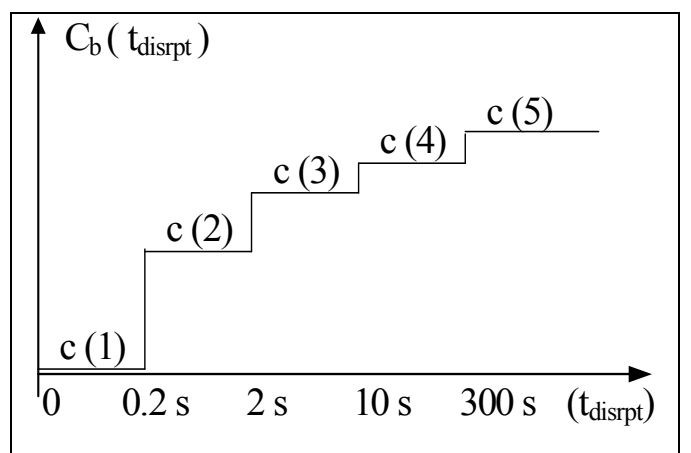

Fig.3.1.b Cost vs. disruption time.

may be much greater and should be decided by the terms of the service contract between the carriers and the service providers that describes the responsibility of the carrier for the service disruption. Finally we express the cost at the data plane in a VTR step $(t \rightarrow t+\Delta t)$ as

$$
C_{D}(t+\Delta t)=C_{b}\left(t_{d i s r p t}\right) \times \sum_{i j} S_{i j}(t) U\left(V_{i j}(t)-V_{i j}(t+\Delta t)\right)
$$

where $t_{\text {disrpt }}$ is the lightpath disruption time due to VTR in the data plane, and $U\left(V_{i j}(t)-V_{i j}(t+\Delta t)\right)$ has been defined as the number of disrupted lighpaths between the nodes $i$ and $j$ in Subsection II.B.2.

\section{Control Plane}

The cost of VTR at the control plane is converted from the blocking probability.

We define the average network revenue in a second as $C_{a}$. If the blocking probability increases from $P_{1}(t)$ to $P_{2}(t)$ during a period of time $T$, the cost can be calculated using an integral expression $\int_{0}^{T} C_{a} \times\left(P_{2}(t)-P_{1}(t)\right) d t$, which represents the revenue loss of the carriers.

According to Equation (4), we have $P_{2} \quad(t)$ $=P_{0}+\left(1-\left(1-\frac{l_{d}(t)}{N \Delta}\right)^{H}\right) e(t), \quad\left(0<t \leq T_{\text {conv }}\right)$ or $P_{0}\left(T_{\text {conv }}<t\right.$ $<\Delta t), P_{l}(t)=P_{0}(0<t<\Delta t)$, and $T=\Delta t$. Then we formulate the cost at the control plane in a VTR step $(t \rightarrow t+\Delta t)$ as

$$
\begin{aligned}
C_{C}(t+\Delta t)= & \int_{t=0}^{T_{\text {conv }}} C_{a} \times\left(P_{0}+\left(1-\left(1-\frac{l_{d}(t)}{N \Delta}\right)^{H}\right) e(t)\left(1-P_{0}\right)-P_{0}\right) d t \\
& +\int_{T_{\text {conv }}}^{\Delta t} C_{a} \times\left(P_{0}-P_{0}\right) d t \\
= & \int_{t=0}^{T_{\text {conv }}} C_{a} \times\left(1-\left(1-\frac{l_{d}(t)}{N \Delta}\right)^{H}\right) e(t)\left(1-P_{0}\right) d t
\end{aligned}
$$

where $l_{d}(t)$ remains constant in each VTR step so that $C_{C}(t+\Delta t)=C_{a} \times\left(1-\left(1-\frac{l_{d}(t)}{N \Delta}\right)^{H}\right)\left(1-P_{0}\right) \int_{t=0}^{T_{c o n v}} e(t) d t$.

We assume that the convergence process of topology discovery in the IP network can be modeled such that 
$\int_{t=0}^{T_{c o n v}} e(t) d t$ is a constant. Note that although the integrand $e(t)$ is not a continuous function, whose value decreases step-wise, the integral still exists. We define $e_{N}(t)$ as the normalized function of $e(t)$ and the constant $C_{e}=$ $\int_{t=0}^{1} e_{N}(t) d t$. Then we get $\int_{t=0}^{T_{\text {conv }}} e(t) d t=T_{\text {conv }} \int_{t=0}^{1} e_{N}(t) d t$ $=T_{\text {conv }} \mathrm{C}_{\mathrm{e}}$, where $C_{e}<1$. Finally the cost function of the control plane is revised as

$$
C_{C}(t+\Delta t)=C_{a} C_{e} T_{c o n v}\left(1-P_{0}\right)\left(1-\left(1-\frac{l_{d}(t)}{N \Delta}\right)^{H}\right)
$$

\section{Integrated Cost Model}

In this subsection we combine all the impact factors of VTR we have discussed. We integrate the performance gain factor together with the cost at both the data and control planes in one cost model.

The original cost of the VTR is expressed as

$$
C_{O}(t+\Delta t)=C_{D}(t+\Delta t)+C_{C}(t+\Delta t) .
$$

$$
\begin{gathered}
C_{O}(t+\Delta t)=C_{b}\left(t_{d i s p t}\right) \times \sum_{i j} S_{i j}(t) U\left(V_{i j}(t)-V_{i j}(t+\Delta t)\right) \\
+C_{a} C_{e} T_{\text {conv }}\left(1-P_{0}\right)\left(1-\left(1-\frac{l_{d}(t)}{N \Delta}\right)^{H}\right)
\end{gathered}
$$

For the purpose of creating a uniform criterion for comparison, we view the performance gain as the negative cost. Hence, using Equation (1) we can define the new cost as

$$
\begin{aligned}
C_{P}(t+\Delta t)=- & \left(\Phi\left(V_{i j}(t+\Delta t), t+\Delta t\right)\right. \\
& \left.-\Phi\left(V_{i j}(t), t+\Delta t\right)\right) \times C_{g}
\end{aligned}
$$

where $C_{g}$ is the multiplier converting the negative of the performance gain into the cost. Then the cost model in a VTR step is described using an integrated expression

$$
\begin{aligned}
& C_{A L L}(t+\Delta t)=C_{P}(t+\Delta t)+C_{O}(t+\Delta t) \\
& =\left(\Phi\left(V_{i j}(t), t+\Delta t\right)-\Phi\left(V_{i j}(t+\Delta t), t+\Delta t\right)\right) \times C_{g} \\
& \quad+C_{b}\left(t_{d i s p p t}\right) \times \sum_{i j} S_{i j}(t) U\left(V_{i j}(t)-V_{i j}(t+\Delta t)\right) \\
& \quad+C_{a} C_{e} T_{\text {conv }}\left(1-P_{0}\right)\left(1-\left(1-\frac{l_{d}(t)}{N \Delta}\right)^{H}\right)
\end{aligned}
$$

Two objectives were proposed in the previous studies $[1,2$, $3,4,5,6,7]$ to measure the performance. The first objective is minimizing the maximum link load to achieve a more balanced load and to reduce congestion. The second is minimizing the average weighted hop count. Respectively we have $\Phi\left(V_{i j}(t), t\right)=-\max _{i, j}\left(\sum_{s, d} \lambda_{i j}^{s d}(t) \Lambda_{i j}(t)\right)$

$$
\text { and } \Phi\left(V_{i j}(t), t\right)=-\frac{1}{\sum_{s, d} \Lambda_{s d}(t)} \sum_{s, d} \sum_{i j} \lambda_{i j}^{s d}(t) \Lambda_{s d}(t)
$$

We adopt the average weighted hop count to compute the performance gain. The practical meaning of $C_{g}$ is the cost of an additional average routing hop for all upper-layer traffic, e.g. IP packets, in the network. We have the revised integrated cost function for each VTR step

$$
\begin{aligned}
& C_{A L L}^{\prime}(t+\Delta t)= \\
& \frac{C_{g}}{\sum_{s, d} \Lambda_{s d}(t+\Delta t)}\left(\sum_{s, d} \sum_{i, j} \lambda_{i j}^{s d}(t+\Delta t)-\sum_{s, d} \sum_{i, j} \lambda_{i j}^{s d}(t)\right) \\
& \quad+C_{b}\left(t_{d i s r p t}\right) \times \sum_{i j} S_{i j}(t) U\left(V_{i j}(t)-V_{i j}(t+\Delta t)\right) \\
& \quad+C_{a} C_{e} T_{\text {conv }}\left(1-P_{0}\right)\left(1-\left(1-\frac{l_{d}(t)}{N \Delta}\right)^{H}\right)
\end{aligned}
$$

\section{A CASE STUdy ON VTR EXECUTION SCHEMES}

In this section we illustrate the application of the established analytical model in studying the VTR algorithms and policies. As a case study, we compare the effect of different VTR execution schemes, which are the special VTR policies defining the time granularity of executing VTR operations. Meanwhile, through this case study, we reveal the impact of two important parameters, the VTR step time and the topology discovery convergence time, on the VTR costs.

\section{A. Fine-Step vs. Coarse-Step VTR Execution Schmemes}

When designing an algorithm to optimize the VTR process, we need to specify the time granularity for this algorithm to be executed. For example, the algorithm can be executed once or ten times per hour. Or we can issue a batch of lightpaths operations for each execution or divide the execution into sequential sub-steps. The VTR execution schemes depend on the VTR algorithms and policies. Some proposed algorithms imply either the batch execution or the sequential execution schemes while others may be adapted to both according to specific VTR policies.

As implied by the branch-exchange algorithm [4], the VTR can be executed via a sequence of reconfiguration operations. At each time only a small number of lightpaths are changed so that the whole virtual topology is changed slowly. Generally we can call such a slowly changing VTR scheme the FineStep VTR execution scheme because the reconfiguration has a finer granularity for each step. For the Fine-Step VTR, the execution frequency is higher, and hence the step time $\Delta t$ is smaller.

The linear formulation based VTR algorithms may be executed less frequently due to the computation complexity [1, 2]. Furthermore, the reconfiguration operations derived from such algorithms are not ordered. They must be executed at the same time to achieve the optimal objective in each step. We call such a VTR scheme, which executes a batch of reconfiguration operations for a relatively long step, the Coarse-Step VTR execution scheme.

The Fine-Step VTR scheme more frequently adapts the virtual topology to the changing traffic demands so as to get more performance gain. On the other hand, frequent reconfiguration means frequent change and may incur higher 
cost. The Coarse-Step VTR scheme may cut down such a cost. The Two-Stage VTR algorithm or two-stage VTR execution scheme is a mixture [3]. It uses a rapid heuristic, bounded on the number of changes, in the reconfiguration stage, which implies a Coarse-Step reconfiguration. Then it optimizes the virtual topology between consecutive traffic changes in the optimization stage, which is more like a Fine-Step VTR scheme.

\section{B. Simlulation and Results}

We conducted our simulation experiments for a network with the physical topology shown in Fig. 4.1.a. The 6-node mesh network has 8 pairs of $2 \mathrm{Gbps}$ fiber links. Each fiber supports two 1 Gbps channels (or wavelengths). Each node has 4 transceivers. The ingress traffic at the six nodes is shown in Fig. 4.1.b. For each experiment, the total integrated cost of the VTR process in the experimental period is calculated as $\sum_{i} C_{A L L}^{\prime}(t+i \Delta t)$, where $C_{A L L}^{\prime}(t+i \Delta t)$ is the revised integrated cost function (defined by Equation (12)) in a VTR step $(t+(i-1) \Delta t \rightarrow t+i \Delta t)$, and the range of $i$ is specified in each experiment.

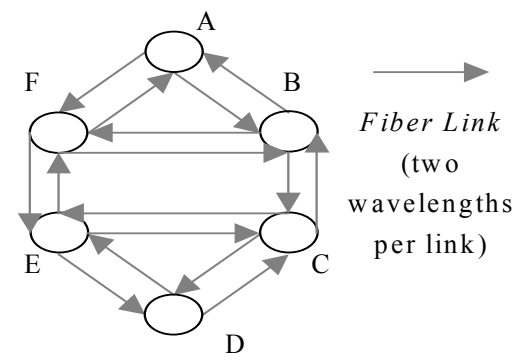

Fig. 4.1.a Physical topology.

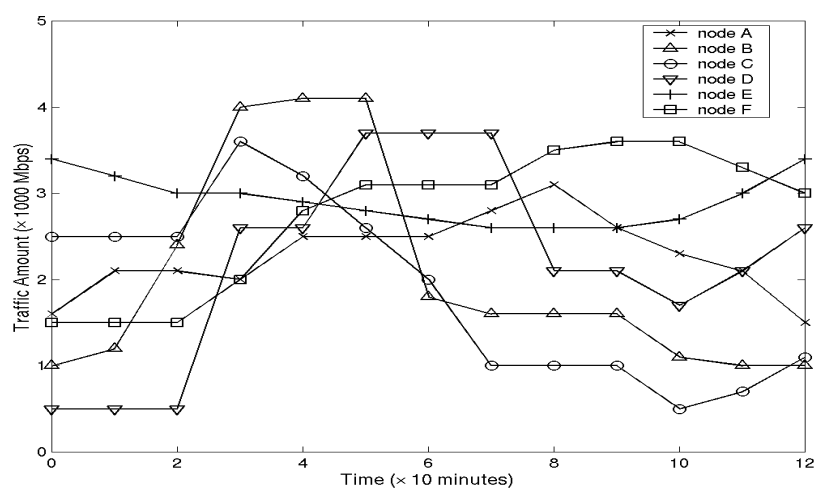

Fig. 4.1.b Traffic at the ingress nodes vs. time.

To reduce the computation complexity, we adapt the pathadd VTR heuristic [3] for both the Coarse-Step and Fine-Step schemes as well as the Two-Stage mixture to simulate the VTR process and obtain the needed cost factors.

We set $P_{0}$ to $0.1, C_{e}$ to $0.4, N \Delta$ to the actual number of lightpaths and $H$ to the actual average weighted hop count in each VTR step. Since it is possible to reduce the VTR disruption time in the data plane to the order of magnitude of tens of milliseconds, as shown in those protection and restoration studies [11], we set $t_{\text {disrpt }}=100 \mathrm{~ms}$. Then, in
$C_{A L L}^{\prime}(t+i \Delta t)$, the lightpath disruption cost $C_{b}\left(t_{\text {disrpt }}\right)$ equals zero.

First of all we study the impact of step time $\Delta t$ and provides a quantitative criterion identifying the Fine-Step and Coarse-Step schemes. We change $\Delta t$ from 1 minute through 60 minutes along the $\mathrm{X}$-axis. Because we do not exactly know the value of $C_{g}$, i.e. the cost paid for each additional average routing hop, we make it proportional to the network revenue in each VTR step time, i.e. $C_{a} \Delta t$, and change it in the range [0.02 $\left.C_{a} \Delta t, 0.2 C_{a} \Delta t\right]$ to guarantee the precision of the experiments, which we will explain later. $T_{\text {conv }}$ is fixed to 10 seconds. The results are shown in Fig. 4.2.

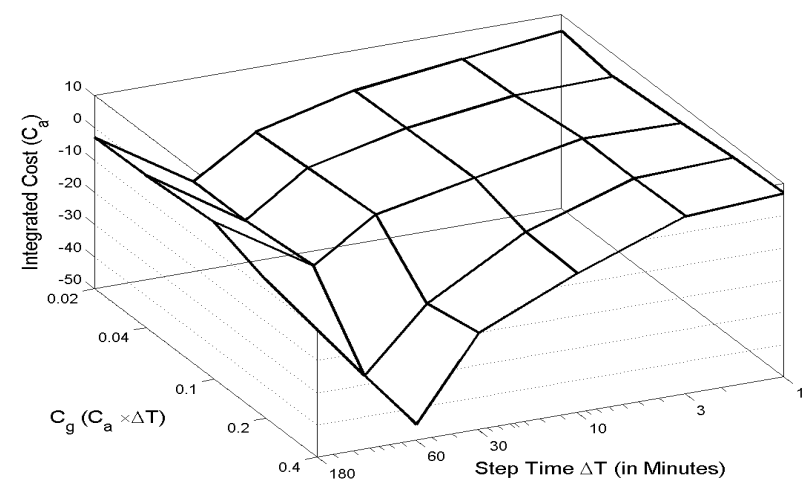

Fig. 4.2 Integrated VTR cost vs. $\Delta t$ and $C_{g}$.

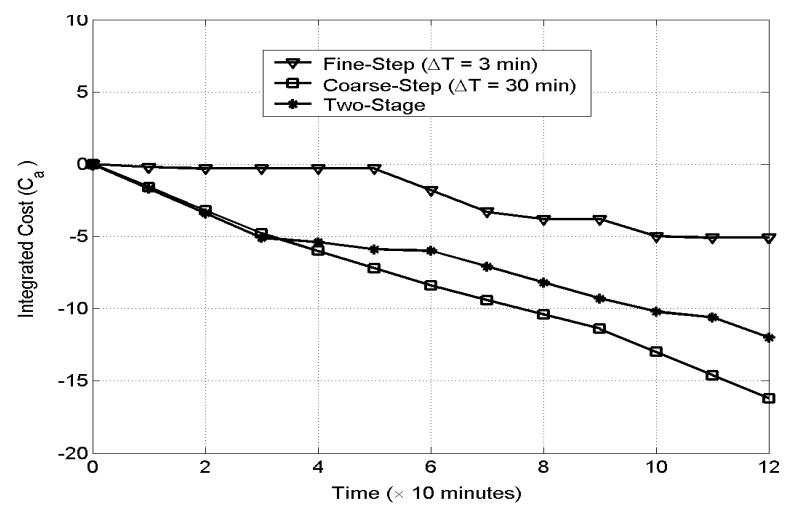

Fig. 4.3 Integrated cost vs. time for different VTR execution schemes.

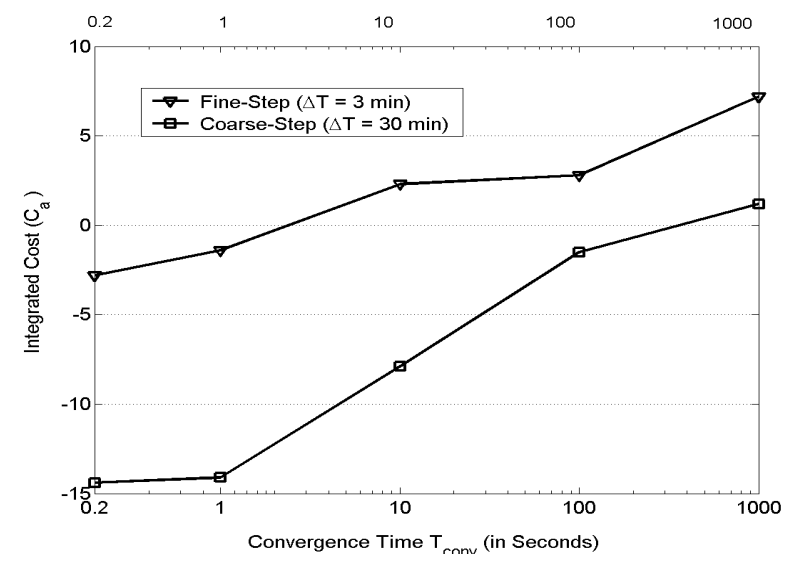

Fig. 4.4 Integrated cost vs. $T_{\text {conv }}$. 
Based on the results above, we set $\Delta t$ to 3 minutes for the Fine-Step VTR scheme and 30 minutes for the Coarse-Step VTR scheme. To experiment on the two-stage scheme, for each 30 minutes, 15 minutes are used for the first stage, and the other 15 minutes are divided into 5 fine steps in the second stage. We fix $C_{g}$ to $0.1 C_{a}$ and $T_{\text {conv }}$ to 10 seconds. The integrated costs accumulated with time for the three schemes are plotted in Fig. 4.3.

We let $T_{\text {conv }}$ exponentially increase from 0.2 second to 1000 seconds along the time axis to further study the sensitivity of the VTR cost model to the convergence time for topology discovery. $C_{g}$ is fixed to $0.1 C_{a} \Delta t$. The results are shown in Fig. 4.4. The two curves represent a fine (3 minutes) and coarse (30 minutes) $\Delta t$ respectively.

\section{Discussion}

In our model, a negative integrated cost means that VTR can be triggered to produce a positive reward. However, computing the precise cost for each of those algorithms depends on the cost parameters in the cost function that need to be customized in specific network situations. An important parameter is $C_{g}$. In our integrated cost model (see Equation (12)) we give it the practical meaning, i.e. the cost resulting from delaying all packets by one hop. Without knowing the actual value of $C_{g}$, we make it a variable in our experiments and justify its range between 0.02 and 0.4 times of the total network revenue in a VTR step time, i.e. $C_{a} \Delta t$.

The step time $\Delta t$ determines the granularity of VTR. According to our experiments, a coarser or larger $\Delta t$ results in less integrated cost. In particular, when $T_{\text {conv }}$ equals 10 seconds, if $\Delta t>6$ minutes, we get a negative cost, i.e. positive reward. The results also show the trend that the reduced cost increases faster than the obtained performance gain when $\Delta t$ increases. However, when $\Delta t \geq 3$ hours, the cost increases, implying that too coarse a step is not desirable.

The topology discovery convergence time $T_{\text {conv }}$ is significant since we use an IP-like topology discovery to control the optical networks. Although the dynamic process of topology discovery may be much shorter than the step time, the results show that its negative impact may still be severe. When $\Delta t=30$ minutes and $T_{\text {conv }}>120$ seconds, i.e. 2 minutes, the integrated cost becomes positive, i.e. unacceptable. The cost model is even more sensitive to $T_{\text {conv }}$ than to $\Delta t$ in this case. According to our experiments, $T_{c o n v}$ should be limited to the order of magnitude of tens of seconds, which is proven possible in [11], to get a positive reward from VTR.

The observations from the experiments show that VTR favors the Coarse-Step VTR execution scheme. The Fine-Step scheme pays much more cost for controlling the network though it gets the performance gain from a more frequent reconfiguration. According to our experiments, the Two-Stage mixture presents a good compromise between the Coarse-Step and Fine-Step schemes.

\section{SUMMARY}

In this study we developed an analytical model to study the impact of VTR on the optical networks, which combine a circuit-based data transport plane with an IP-like control plane. Our models identify and analyze the impact factors from both the data and control planes independent of any specific VTR algorithm or policy. This allows the carriers to choose a VTR algorithm or policy adaptively according to real-time network situations, instead of being bound to a fixed algorithm or policy. We integrated these factors into a uniform cost model. This cost model uses the average network revenue per second as its unit and provides a practical and precise criterion for carriers to compare different VTR algorithms and policies and decide the conditions for triggering the VTR operations.

We conducted a case study on the special VTR policies, i.e. the execution schemes, using our analytical models. An interesting finding is that the Coarse-Step execution scheme has a lower cost than the Fine-Step execution scheme. Through this case study we also showed that VTR step time and topology discovery convergence time are two important parameters though ignored by many previous studies.

We plan to improve our models by deriving a more sophisticated blocking probability model to extend the simplified one in Subsection II.C.3. Case studies with longer lightpath disruptions and more complex network topologies will also be made to provide further evaluations.

\section{REFERENCES}

[1] D. Banerjee and B. Mukherjee, "Wavelength-routed Optical Networks: Linear Formulation, resource Budget Tradeoffs and a reconfiguration Study", IEEE/ACM Transactions on Networking, vol.8, no.5, pp.598607, Oct. 2000.

[2] B. Ramamurthy and A. Ramakrishnan, "Virtual Topology Reconfiguration of Wavelength-routed Optical WDM Networks", in Proceedings of IEEE Globecom 2000, San Francisco, CA, Nov. 2000.

[3] N. Sreenath, C. Siva Ram Murthy, "A Two-Stage Approach for Virtual Topology Reconfiguration of WDM Optical Networks", Optical Networks Magazine, May/Jun., 2001.

[4] A.S. Acampora, J.-F. P. Labourdette and G. W. Hart, "Branch-Exchange Sequences for Reconfiguration of Lightwave Networks," IEEE Transactions on Communications, Vol. 39, No. 10, pp. 2822-2832, Oct., 1994.

[5] E. Baldine and G. N. Rouskas, "Reconfiguration and Dynamic Load Balancing in Broadcast WDM Networks," Photonic Networks Communications Journal, vol. 1, pp. 49-64, Jun. 1999.

[6] E. Baldine and G. N. Rouskas, "Dynamic Reconfiguration Policies for WDM Networks", in Proceedings of IEEE Infocom 1999, New York, NY, Mar. 1999.

[7] G. N. Rouskas and M. H. Ammar, "Dynamic Reconfiguration in Multihop WDM Networks," Journal of High Speed Networks, vol. 4, no. 3, pp. 221--238, Jun. 1995.

[8] A. Banerjee et al, "Generalized Multiprotocol Label Switching: An Overview of Routing and Management Enhancement", IEEE Communications Magazine, Jan. 2001.

[9] J. Schallenberg, "Is 50 ms Restoration Necessary? ", IEEE Bandwidth Management Workshop, 2001.

[10] J. Sosnosky, "Service applications for SONET DCS distributed restoration", IEEE JSAC Vol 12, No. 1. Jan. 1994.

[11] J. Y. Wei, et. al, "IP over WDM Network Traffic Engineering Demonstration and Experimentation", in Proceedings of OFC 2001, Anaheim CA, Mar. 2001.

[12] B.Mukherjee, D. Banerjee, S.Ramamurthy, A.Mukherjee, "Some Principles for Designing a Wide-Area WDM Optical Network", IEEE Transactions on Networking, Vol.4, n.5, pp. 684-695, Oct. 1996.

[13] C. Labovitz, R. Wattenhofer, S. Venkatachary, and A. Ahuja, "The Impact of Internet Policy and Topology on Delayed Routing Convergence," in Proceedings of IEEE INFOCOM 2001, Anchorage, AK, Apr. 2001. 